\title{
Classifying the Electronic and Optical Properties of Janus Monolayers
}

Riis-Jensen, Anders Christian; Deilmann, Thorsten; Olsen, Thomas; Thygesen, Kristian Sommer

Published in:

ACS Nano

Link to article, DOI:

10.1021/acsnano.9b06698

Publication date:

2019

Document Version

Peer reviewed version

Link back to DTU Orbit

Citation (APA):

Riis-Jensen, A. C., Deilmann, T., Olsen, T., \& Thygesen, K. S. (2019). Classifying the Electronic and Optical Properties of Janus Monolayers. ACS Nano, 13(11), 13354-13364. https://doi.org/10.1021/acsnano.9b06698

\section{General rights}

Copyright and moral rights for the publications made accessible in the public portal are retained by the authors and/or other copyright owners and it is a condition of accessing publications that users recognise and abide by the legal requirements associated with these rights.

- Users may download and print one copy of any publication from the public portal for the purpose of private study or research.

- You may not further distribute the material or use it for any profit-making activity or commercial gain

- You may freely distribute the URL identifying the publication in the public portal

If you believe that this document breaches copyright please contact us providing details, and we will remove access to the work immediately and investigate your claim. 


\title{
Classifying the Electronic and Optical Properties of Janus Monolayers
}

\author{
Anders C. Riis-Jensen, ${ }^{*} \dagger$ Thorsten Deilmann, ${ }^{*}, \dagger$ Thomas Olsen $^{\dagger}{ }^{\dagger}$ and Kristian S. \\ Thygesen $^{\dagger}$ \\ $\dagger C A M D$, Department of Physics, Technical University of Denmark, DK-2800 Kongens \\ Lyngby, Denmark \\ $\ddagger$ Institut für Festkörpertheorie, Westfälische Wilhelms-Universität Münster, 48149 \\ Münster, Germany \\ - Center for Nanostructured Graphene (CNG), Technical University of Denmark, DK-2800 \\ Kongens Lyngby, Denmark \\ E-mail: achrii@fysik.dtu.dk; thorsten.deilmann@wwu.de
}

\section{Abstract}

Inspired by the recent synthesis of monolayer MoSSe we conduct a first-principles highthroughput investigation of 216 MXY Janus monolayers consisting of a middle layer of metal atoms (M) sandwiched between different types of chalcogen, halogen, or pnictogen atoms $(X, Y)$. Using density functional theory and many-body perturbation theory we perform an exhaustive computational characterization of the 70 most stable semiconducting monolayers. These are found to exhibit diverse and fascinating properties including finite outof-plane dipoles, giant Rashba-splittings, direct and indirect band gaps ranging from 0.7 to 3.0 $\mathrm{eV}$, large exciton binding energies, and very strong light matter interactions. The data has been generated using the workflow behind the Computational 2D Materials Database (C2DB) and is freely available online. Our work expands the class of known Janus monolayers and points to several potentially synthesisable structures, which could be interesting candidates for valleyor opto-electronic applications, or for generating out-of-plane electric fields to control charge transfer, charge separation, or band alignments in van der Waals heterostructures.
Two-dimensional (2D) semiconductors composed of a single or few layers of covalently bonded atoms exhibit interesting physical properties including layer-dependent band gaps, spin-valley coupling, $\stackrel{314}{,}$ and strong light-matter interactions ${ }^{5[6]}$ dominated by significant excitonic effects. ${ }^{711}$ The extreme thinness of these materials make them highly susceptible to their environment opening up for easy tunability of their properties via strain, $\frac{12113}{,}$ external fields, ${ }^{\sqrt{14}} \sqrt{16}$ or by varying the dielectric environment. 17118 The possibility of stacking different 2D layers into van der Waals heterostructures opens further prospects for designing artificial structures with tailored properties. $\frac{19120}{}$

While the field of 2D materials has long been dominated by graphene, boron-nitride, and the transition metal dichalcogenides (TMDCs), more recently other types of materials have caught the attention of the community. These include the MXenes (metal-nitrides and carbides), ${ }^{21}$ metal halides such as the ferromagnetc $\mathrm{CrI}_{3},{ }^{22}$ and the monolayers MoSSe and BiTeI with finite out-of-plane dipole moments,,$\sqrt{23} \sqrt[25]{2}$ which are the subject of the current paper. To date more than 50 materials have been synthesized in monolayer form ${ }^{26}$ but computational studies suggest that many hundreds of known layered bulk crystals can be exfoli- 
ated to the single layer limit. $27 \sqrt[29]{29}$ One such database of $2 \mathrm{D}$ materials is the Computational 2D Materials Database (C2DB), based on a comprehensive workflow for characterizing the stability and basic properties of hypothetical monolayers, was recently launched. 26

Ferroelectric materials are interesting for a number of reasons. For example, the internal electric field in such materials can lead to a staggered band gap profile producing $2 \mathrm{D}$ confined electron gasses at the surface of a ferroelectric insulator ${ }^{30131}$ or it could be used to separate electron-hole pairs in photovoltaic systems. ${ }^{32[33}$ In bulk crystals, the spontaneous polarization is typically driven by the displacement of an ion away from a high-symmetry position. Due to the relatively small energy gain associated with this symmetry breaking, conventional ferroelectrics lose their polarization above a certain phase transition temperature and become paraelectric. In contrast, in the recently synthesised MoSSe and the exfoliated BiTeI monolayers, the finite dipole is not associated with a spontaneous symmetry breaking but is a property of the structure independent of temperature. Theoretical studies have indicated that Janus monolayers could be useful as structural phase transition materials, $\underline{34}$ for photo-catalytic water splitting,, 35 and for combined in-plane/out-of-plane piezoelectricity. In addition, they could facilitate a number of interesting effects when stacked into multilayers or combined with other 2D materials. For example, the large intrinsic dipole could be used to separate intralayer excitons into interlayer excitons, ${ }^{37}$ move charges between different layers of a heterostructure, $\stackrel{38 \mid 39}{ }$ or tune band alignment and Schottky barriers at interfaces.

In this paper we perform a systematic investigation of MXY Janus structures in the crystal structures of MoSSe (H-phase) and BiTeI (Tphase). Following the workflow developed for the $\mathrm{C} 2 \mathrm{DB}$ we first evaluate the thermodynamic and dynamic stability of the materials and next compute the magnetic, elastic, electronic, and optical properties of the 70 most stable materials exhibiting a finite band gap. Due to the known limitations of density functional theory (DFT) we employ the $\mathrm{G}_{0} \mathrm{~W}_{0}$ self-energy method to obtain quasiparticle band structures and the Bethe-Salpeter Equation (BSE) for optical excitations. We provide an overview of our results including a discussion of some structureproperty relations, a classifications of the materials' electronic properties, and a more in-depth description of a few representative materials. All results are available in the $\mathrm{C} 2 \mathrm{DB}$ and can be browsed online or directly downloaded.

\section{Results}
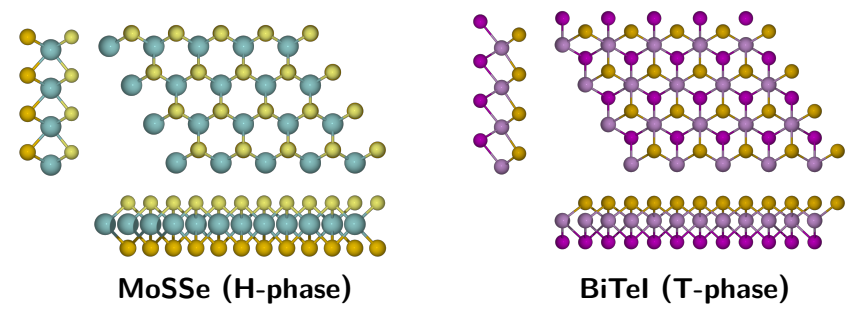

Figure 1: The two types of MXY Janus monolayers considered in this work: MoSSe (left) and BiTeI (right).

We consider MXY Janus monolayers in the crystal structures of the experimentally realized $\mathrm{MoSSe}^{23}$ and BiTeI ${ }^{25}$ monolayers, respectively. The two structures are shown in Fig. 1. Due to the lack of mirror symmetry, such structures possess a macroscopic dipole moment in the out-of-plane direction. The size of the dipole will depend on various factors, but as we will see it correlates strongly with the difference in electronegativity of the $\mathrm{X}$ and $\mathrm{Y}$ atoms.

Hypothetical MXY Janus monolayers are produced by decorating the MoSSe and BiTeI prototype crystal structures. For the central M atom we consider the group IV-V transition metal atoms (Ti, Zr, Hf, V, Nb, Ta, Cr, Mo, $\mathrm{W})$ as well as the pnictogens (As, Sb, Bi). For the $\mathrm{X}$ and $\mathrm{Y}$ atoms we consider the chalcogens ( $\mathrm{S}, \mathrm{Se}, \mathrm{Te})$, halogens $(\mathrm{Cl}, \mathrm{Br}, \mathrm{I})$, and the pnictogens (As, Sb, Bi). This yields a total of 108 monolayers in each of the two crystal structures. The complete set of considered structures can be deduced from Figs. 2 and 3 . For these 216 monolayers, various electronic and optical properties are calculated (see methods section and fig. 13.). 


\section{Stability}

We assess the thermodynamic stability of the monolayers from their energy above the convex hull, $E_{\text {hull }}$. The convex hull is a phase diagram representing the energy of the most stable (possibly mixed-) phase of the material as a function of the stoichiometry. If a hypothetized material lies above the convex hull, i.e. if $E_{\text {hull }}>0$, the material is thermodynamically unstable and will eventually decompose into other phase(s), if it can be synthetized at all. In the current work, the convex hull is constructed from the 2836 most stable binary bulk compounds obtained from the OQMD database. $\stackrel{40}{ }$ The total energy of these bulk structures were calculated with the PBE exchange-correlation functional using GPAW code with the same settings as applied for the Janus monolayers. The calculated energy above convex hull per atom, $E_{\text {hull }}$, for the 216 MXY Janus monolayers is shown in Figs. 2 and 3.

In general, the variation in $E_{\text {hull }}$ with the type of atoms is much larger than the difference between the two crystal phases, i.e. for a given MXY composition, the difference in energy between the two phases is relatively small. The most stable structures are found for: (1) Hphase with $\mathrm{M}$ a group V-VI transition metal and both $\mathrm{X}$ and $\mathrm{Y}$ a chalcogen. (2) H-phase with $\mathrm{M}$ a group IV transition metal and both $\mathrm{X}$ and $\mathrm{Y}$ a halogen. (3) T-phase with $\mathrm{M}$ a pnictogen and $\mathrm{X} / \mathrm{Y}$ a chalcogen/halogen. (4) T-phase with $\mathrm{M}$ a group IV-V transition metal and both $\mathrm{X}$ and $\mathrm{Y}$ a chalcogen.

To test for dynamical stability we calculate the elastic coefficients and the phonons at the $\Gamma$-point and the corner points of the BZ. Only materials with positive elastic coefficients and no imaginary phonon frequencies are considered stable.

The combined criteria of $E_{\text {hull }}<0.2 \mathrm{eV}$, real phonon freqencies (at high symmetry points) and positive elastic constants, reduces the original set of 216 materials to 93 . Out of these 70 are non-magnetic and have a finite PBE band gap. These materials are listed in Tables 1 and 2 in appendix A (see supporting information online), where we list various properties such
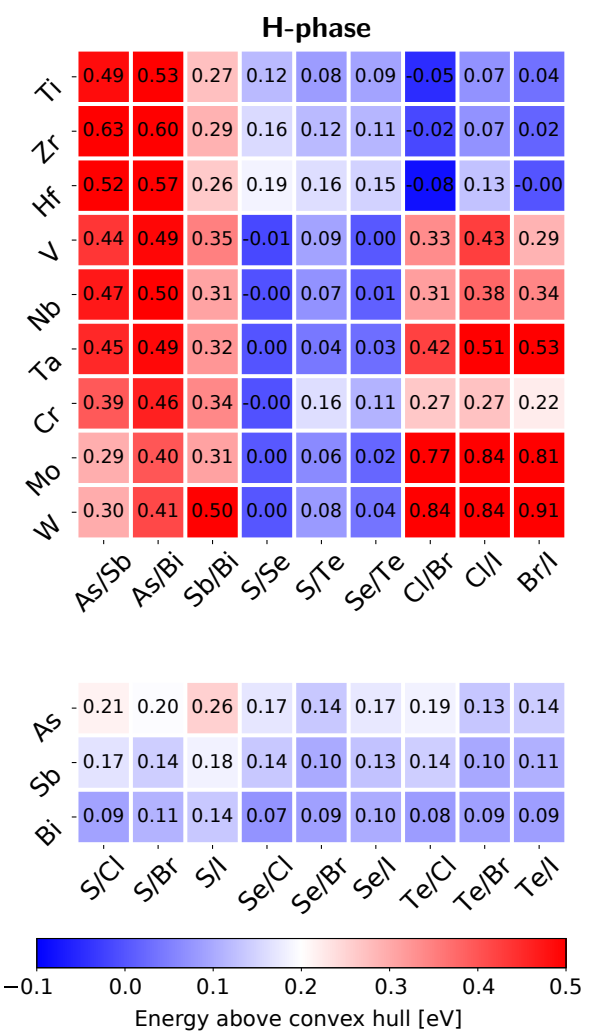

Figure 2: Thermodynamic stability of the $\mathrm{H}-$ phase for different MXY Janus monolayers. The colors denote the energy above the convex hull in $\mathrm{eV} /$ atom.

as PBE, HSE, and GW band gaps, electron and hole effective masses, BSE exciton binding energies, and the vacuum level shift across the monolayers. These 70 monolayers will be discussed further in the following.

\section{Out-of-plane dipole}

As already mentioned the lack of mirror symmetry in the Janus structures leads to a finite out-of-plane dipole moment. Because of the difference in electronegativity between the $\mathrm{X}$ and $\mathrm{Y}$ atoms, charge will be transferred across the metal layer creating an out-of-plane dipole moment. The size of the dipole can be quantified by the shift in the vacuum level on the two sides of the layer, see Figure 4 . We note that because of the dipole-induced potential step, the vacuum level on the two sides of the monolayer will in general be different. We choose to reference the band edge energies to the average value of the asymptotic potential on the two sides of 

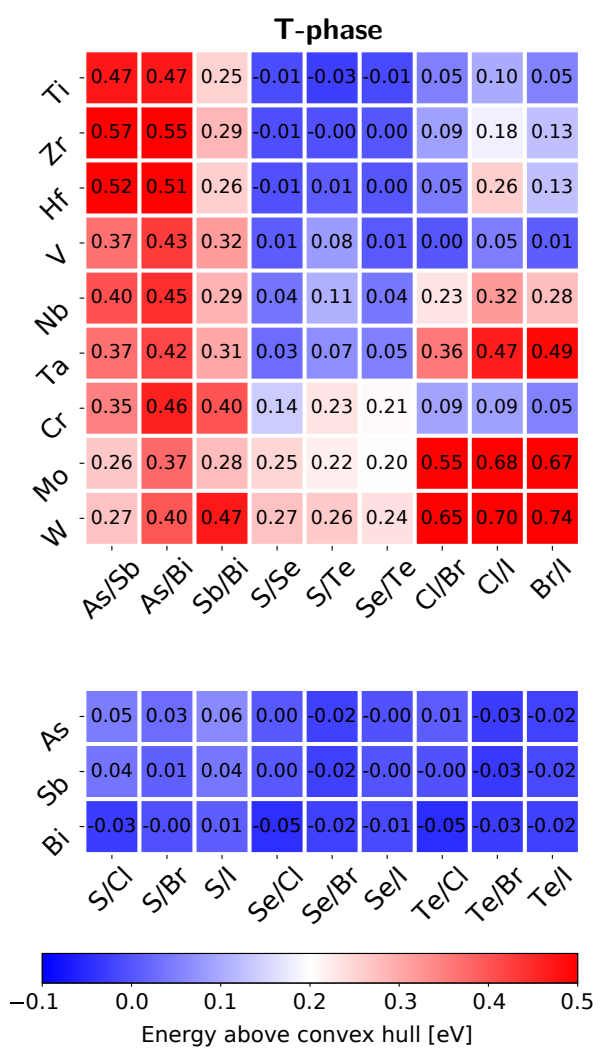

Figure 3: Same as Fig. 2 but for the T-phase.

the monolayer. Combined with the size of the potential step (vacuum level shift) this allows to obtain the band energies relative to the vacuum level on either side of the monolayer. Not unexpectedly, the size of the dipole (or equivalently the vacuum level shift) correlates with the difference in electronegativity of the $\mathrm{X}$ and $\mathrm{Y}$ atoms $(\Delta \chi)$, see Figure 5. Pronounced exceptions from this trend occur for e.g. ZrSeTe and $\mathrm{ZrSTe}$, which have very similar vacuum level shift despite a significant $\Delta \chi$ of 0.5 . These deviations suggest that other attributes such as interatomic distances, the electronegativity of the $\mathrm{M}$ atom, and the specific interatomic wave function overlaps, also influence the size of the resulting dipole.

Figure 5 also reveals a wide range of vacuum level shifts possessed by the Janus monolayers, ranging from essentially $0 \mathrm{eV}$ to around $1.8 \mathrm{eV}$. The wide range of dipoles make the Janus monolayers ideal for applications in van der Waals heterostructure-based devices where they could be used to set up internal fields of varying strength to control charge transfer, charge separation, and band alignment.

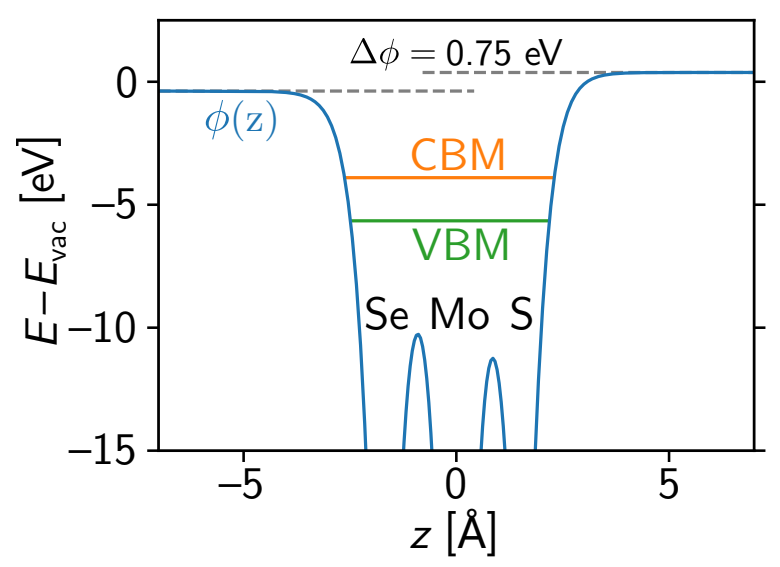

Figure 4: Averaged potential, $\phi(z)$, (blue) compared to the vacuum level for H-MoSSe. Between $+z$ and $-z$ a jump in the potential of around $0.75 \mathrm{eV}$ is observed as a consequence of the out-of-plane dipole moment. In green and orange the valence band maximum and the conduction band minimum are indicated.

In the following two sections we provide an overview of the electronic and optical properties of the 70 stable and semiconducting MXY Janus monolayers identified in the previous section. By necessity the discussion will focus on general trends and classifications of the qualitative features. A few band structures and optical spectra will be discussed for specific representative materials, and we refer to interested reader to the $\mathrm{C} 2 \mathrm{DB}$ where all data is available.

\section{Electronic band structures}

Before focusing on the 70 stable semiconductors, we give an overview of the band structure of all the 216 Janus monolayers (excluding the few magnetic compounds). By manual inspection, we have found that the qualitative features of the PBE band structures, with very few exceptions, can be classified according to the group of the $\mathrm{M}$ and $\mathrm{X} / \mathrm{Y}$ elements as shown in Fig. 6. The plot is divided into the H-phase (top) and the T-phase (bottom). Notice that the bottom rows refer to structures with mixed chalcogens and halogens for the XY atoms, i.e. a chalcogen on one side on the $\mathrm{M}$ layer and a halogen on the other side. The matrix plot first of all shows whether the mono- 


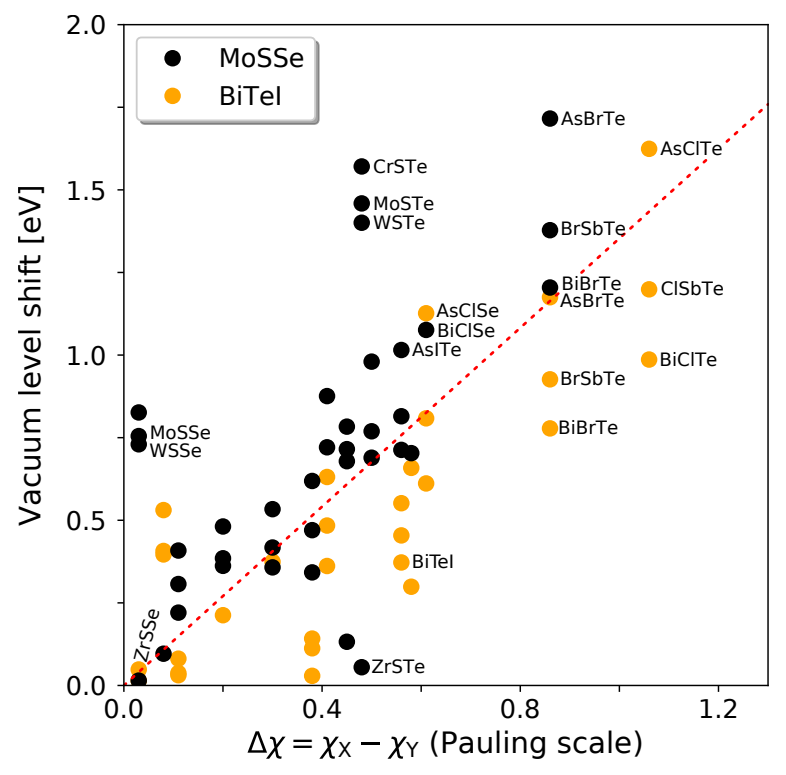

Figure 5: Correlation between the electronegativity difference of $\mathrm{X}$ and $\mathrm{Y}$ and out-of-plane dipole quantified by the vacuum level shift (see Fig. 4). The results for the H-phase (T-phase) are shown in black (orange), the red line is linear fit with a slope of about $1.35 \mathrm{eV} / \Delta \chi$ (Pauling scale).

layer is metallic or semiconducting for a certain combination of $\mathrm{M}$ and XY atoms. For the semiconductors, it is further indicated whether the valence band maximum (VBM) and conduction band minimum (CBM) are located at a highsymmetry point or a high-symmetry path. Materials belonging to the last row are found to exhibit a Rashba split conduction band while H-phase monolayers with group VI metals in combination with $\mathrm{S}$ and $\mathrm{Te}$ are found to exhibit a Rashba split valence band maximum (see below for a more detailed discussion). The classification holds for the vast majority of materials, with a few exceptions that we return to below.

The greater part of 216 monolayers are found to be metallic. On the other hand, among the 93 stable materials only 23 are metals (7 in the $\mathrm{H}$-phase and 16 in the T-phase among which 7 are ferromagnetic) while 70 are semiconducting. The semiconducting structures in the H-phase are found among four combinations of $\mathrm{M}$ and $\mathrm{XY}$ atoms while T-phase structures are semiconducting for only two combinations of $\mathrm{M}$ and $\mathrm{XY}$ atoms. A direct band gap is only found
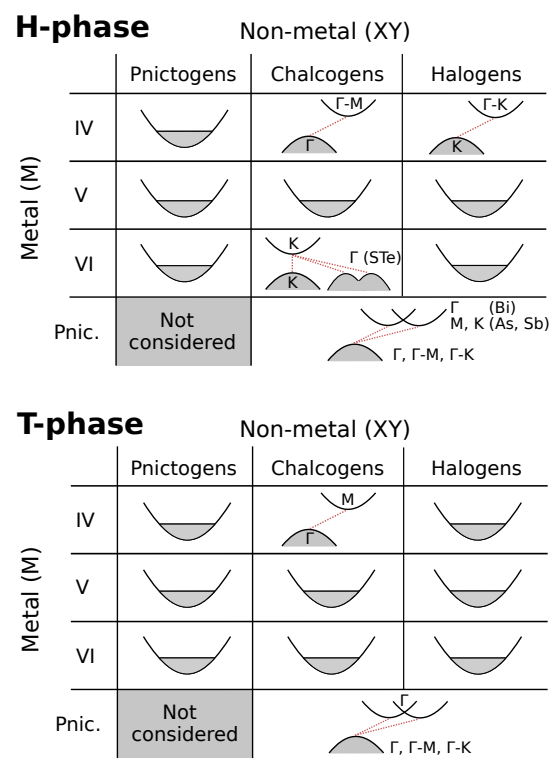

Figure 6: Qualitative classification of the band structure of MXY Janus monolayers in the $\mathrm{H}$ phase (top) and T-phase (bottom). We classify the electronic properties of the MXY monolayers by if they are found to be metallic or semiconducting. For the semi-conducting materials we further state at which high symmetry point or high symmetry path the band extrema are located. This is done based on the combination of $\mathrm{M}$ (vertical axis) and XY (horizontal) atoms. The classification is based on the PBE band structure omitting the few magnetic materials.

for the H-phase when the $\mathrm{M}$ atom is a group VI transition metal atom $(\mathrm{Cr}, \mathrm{Mo}, \mathrm{W})$ and XY are chalcogen atoms, except for structures of the form MSTe. A close to direct band gap is also found in a few cases, namely for structures where $\mathrm{M}$ is a pnictogen atom (last row in Fig. 6). These structures are, however, subject to a considerable Rashba splitting close to the CBM and in the H-phase the position of CBM depends on the nature of the $\mathrm{M}$ atom.

There are a few exceptions to the classification found in Fig. 6. In both the $\mathrm{H}$ - and $\mathrm{T}$ phase with a group IV transition metal atom and X,Y both chalcogen atoms, some of the structures are found to be semi-metallic with PBE but open a band gap with the HSE functional. These structures are H-HfSTe, H-TiSTe, 
H-TiSeTe, T-HfSTe, T-HfSeTe, and T-ZrSTe. When the HSE functional is used, the band structures follow the classification in Fig. 6. Because the workflow classifies the materials as metals/non-metals on the basis of the PBE band structure (see Fig. 13), these materials are treated as metals and consequently the $\mathrm{G}_{0} \mathrm{~W}_{0}$ band structure and BSE absorption spectrum have not been calculated. T-TiSTe and T-TiSeTe are predicted to be semi-metallic by both PBE and HSE.

\section{Rashba splitting}

The Rashba effect is a momentum-dependent splitting of the spin band structure in the vicinity of a band extremum. It occurs as a result of spin-orbit coupling in systems lacking an inversion center, most notably in 2D systems with broken mirror symmetry such as surfaces, interfaces, or 2D Janus structures, but has also been observed in bulk crystals such as the lay-

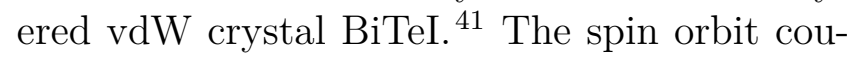
pling leads to a splitting of the spin bands by the effective magnetic field, $\mathbf{v} \times \mathbf{E} / c^{2}$, seen by an electron moving with velocity $\mathbf{v}$ in an electric field, $\mathbf{E}(\mathbf{r})$. In reality, the total electric field has a complex spatial variation on a subatomic length scale. However, the prevailing (over)simplified picture of the Rashba effect, neglects the microscopic details of $E$ and simply replaces it by a constant out-of-plane polarized field. While this model captures the qualitative features of the Rashba splitting we show below that it is completely unable to provide a quantitative description.

Close to an (isotropic) band extremum, the Rashba-split spin bands take the form

$$
\varepsilon_{n k}=\frac{\hbar^{2}}{2 m^{*}} k^{2} \pm \alpha k
$$

where $\alpha$ is the Rashba coupling parameter which quantifies the strength of the effect. The Rashba coupling parameter is related to the energy- and momentum-shift of the spin bands via $\alpha=2 E_{\mathrm{R}} / k_{\mathrm{R}}$. In tables I and II in the supplementary information we list $E_{\mathrm{R}}$ and $k_{\mathrm{R}}$ for the Janus monolayers where a notable Rashba

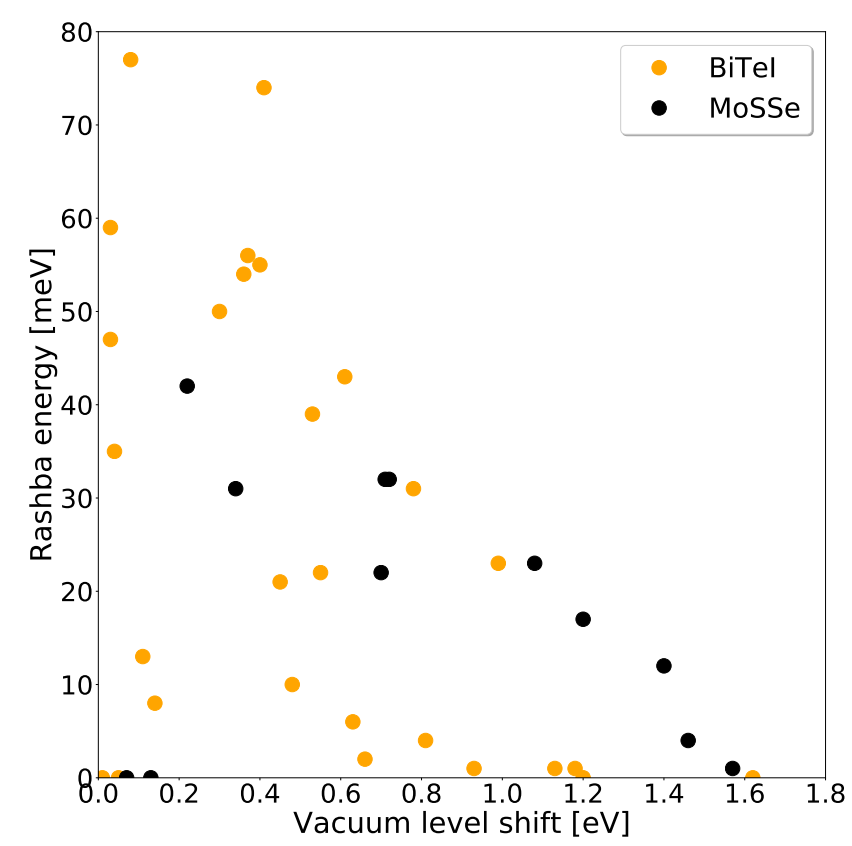

Figure 7: The vacuum level shift ( $x$-axis) against the Rashba energy ( $y$-axis).

splitting is found at either the VBM or CBM. We find values up to $77 \mathrm{meV}$ for $E_{\mathrm{R}}$ and 82 $\mathrm{m} \AA^{-1}$ for $k_{\mathrm{R}}$. For monolayer BiTeI we find $E_{\mathrm{R}}=56 \mathrm{meV}$, which is about half of the measured value of bulk BiTeI. 41 For the VBM we find Rashba coupling parameters in the range 5$30 \mathrm{meVA}$ in good agreement with previous calculated values for H-phase TMDC Janus structures. $\stackrel{42}{ }$ For the CBM we find similar values for most monolayers and much larger values for some monolayers (e.g.: T-AsISe, T-BiISe, TBiITe, T-SbISe, T-SbIS, and T-SbITe) ranging up to some hundreds meVA.

In fig. 7 we show that there is absolutely no correlation between the Rashba energy, $E_{\mathrm{R}}$, and the size of the vacuum level shift. The latter is proportional to the out-of-plane dipole and thus the averaged out-of-plane electric field inside the monolayer. This clearly demonstrates the quantitative limitations of the simple Rashba model. Furthermore, in Fig. 1 in the supporting information we show that there is only very weak correlation between $E_{\mathrm{R}}$ and the strength of the spin-orbit coupling (here quantified by the shift of the band edge energy induced by the spin-orbit coupling). These findings underlines the complex nature of the Rashba effects. 

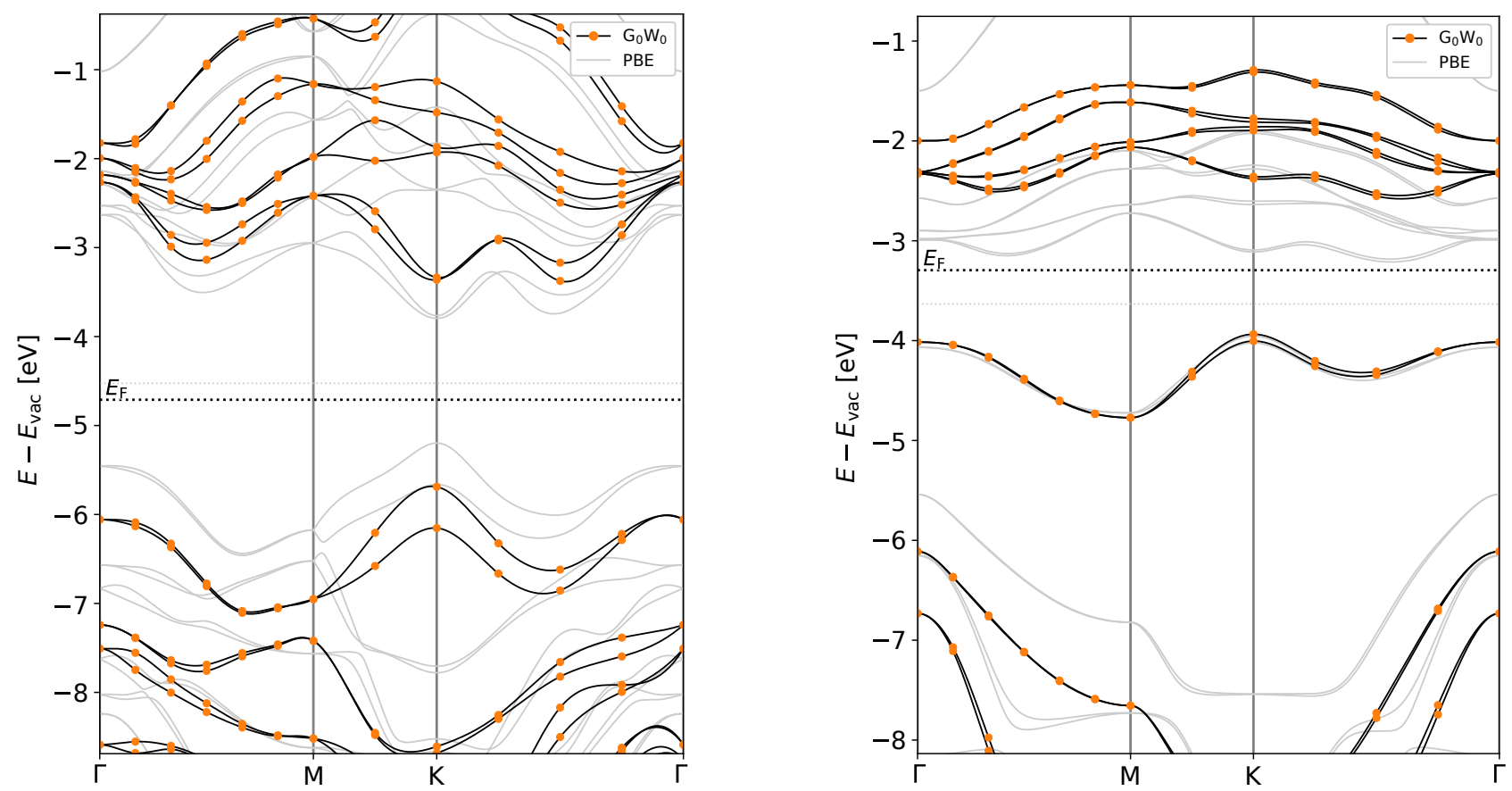

Figure 8: Band structure of H-WSSe (left panel) and H-TiClI (right panel). All energies are referenced to the vacuum level defined as the average of the asymptotic potential on the two sides of the monolayer, see Fig. 4 . The orange dots and black interpolated lines show the $\mathrm{G}_{0} \mathrm{~W}_{0}$ results while the grey lines show the PBE result.

\section{Examples of band structures}

As representatives for $\mathrm{H}$-phase group VIchalcogen and group IV-halogen MXY monolayers we show in Fig. 8 the band structures of H-WSSe (left panel) and H-TiClI (right panel). We note on passing that all band structures include spin-orbit coupling. The band structure of WSSe is similar to the well known TMDCs while TiClI hosts a characteristic flat valence band separated from lower-lying occupied bands. Our PBE results (grey lines) are compared to $\mathrm{G}_{0} \mathrm{~W}_{0}$ (orange symbols) and the band energies are aligned to the vacuum level defined as the average of the vacuum level on the two sides of the layer. As expected, we find a distinct opening of the gaps in $\mathrm{G}_{0} \mathrm{~W}_{0}$ emphasizing the previously found important role of many-body effects in $2 \mathrm{D}$ materials. $\frac{5143}{1}$ For WSSe the direct gap at $\mathrm{K}$ opens from $1.40 \mathrm{eV}$ (PBE) to $2.33 \mathrm{eV}\left(\mathrm{G}_{0} \mathrm{~W}_{0}\right)$.

While $\mathrm{G}_{0} \mathrm{~W}_{0}$ produces an almost symmetric shift in opposite directions of the occupied and unoccupied states in WSSe the situation is very different for TiClI where the intermediate occupied band at around $-4 \mathrm{eV}$ is almost unchanged by the $\mathrm{G}_{0} \mathrm{~W}_{0}$ self-energy. We speculate that this is a result of the orbital character of the intermediate band wave function, which contains almost equal amounts of $\mathrm{Cl} / \mathrm{I} p$ states, which are mostly occupied, and Ti $d$ states, which are mostly unoccupied.

In Fig. 9 we show the band structure of BiClTe in the T-phase. Both PBE and $\mathrm{G}_{0} \mathrm{~W}_{0}$ predicts a direct gap of 0.64 and $1.74 \mathrm{eV}$, respectively. However, in contrast to the H-phase group VI-chalcogens discussed above, the direct gap resides at $\Gamma$ rather than K. Furthermore, we note that the $\mathrm{G}_{0} \mathrm{~W}_{0}$ correction to the $\mathrm{PBE}$ bands is rather asymmetric: While the valence bands shift down by about $0.9 \mathrm{eV}$ the conduction bands shift up by only $0.3 \mathrm{eV}$. While the valence band at $\Gamma$ is doubly degenerate with light and heavy hole masses of $0.24 m_{0}$ and $0.31 m_{0}$, respectively, the conduction band has a Mexican hat-like shape due to a Rashba effect driven by the combination of strong spin-orbit coupling and lack of inversion symmetry. This behavior is not reproduced by the $\mathrm{G}_{0} \mathrm{~W}_{0}$ calculation due to the interpolated nature of the band structure. 


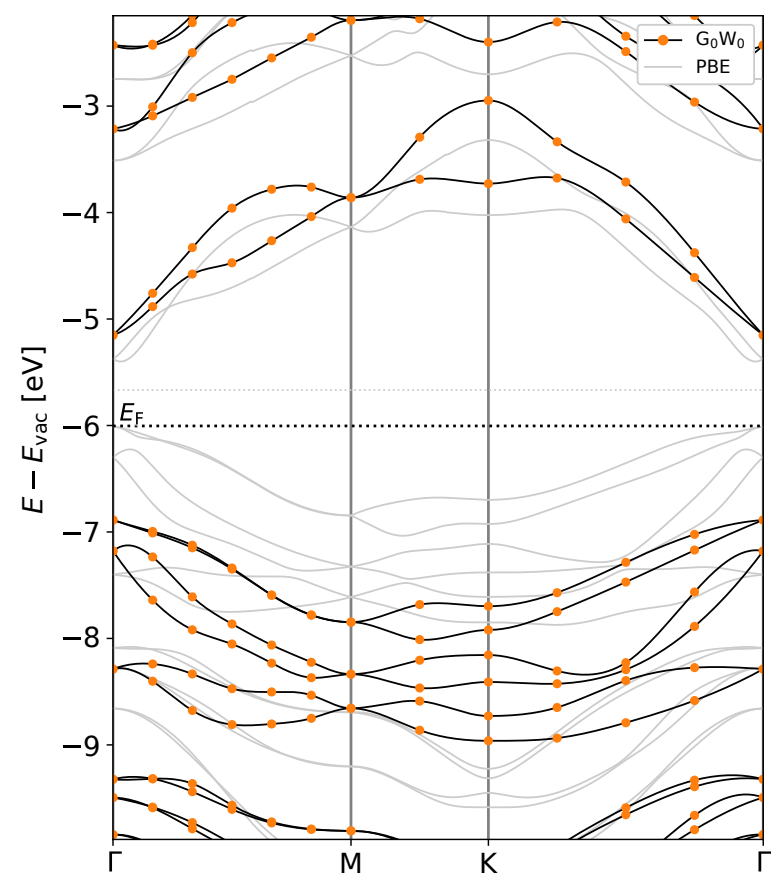

Figure 9: Same as in Fig. 8, but for T-BiClTe.

Fig. 10 shows the distribution of the $\mathrm{G}_{0} \mathrm{~W}_{0}$ band gaps of all 70 MXY monolayers. As is the case for the out-of-plane dipole, the band gap size shows rather large variation ranging from $0.7 \mathrm{eV}$ to $3.0 \mathrm{eV}$.

\section{Optical absorption and exci- tons}

One of the most distinctive characteristics of $2 \mathrm{D}$ semiconductors is their strong interaction with light. ${ }^{44}$ This effect arises from the localized nature of the electronic states in one direction (quantum confinement) in combination with the weak dielectric screening, which leads to strongly bound electron-hole pairs (excitons) with large oscillator strengths. ${ }^{556}$ Specifically for the TMDCs, the optical properties also include spin-valley selective excitation of electron-hole pairs by means of circularly polarized light. ${ }^{3 / 4}$ However, exploitation of this feature, e.g. for valleytronics, $\frac{45}{4}$ is currently limited by inter-valley scattering that reduces the lifetime and mean free path of valleylabelled excitons. Due to the similarity with the TMDCs, both in terms of atomic and electronic structure, some of the MXY Janus mono- layers might also display spin-valley coupling and thus could become interesting candidates for valleytronics applications.

Here we explore some basic optical and excitonic properties of the 70 stable, semiconducting MXY Janus monolayers. Specifically, we calculate the optical absorbance spectrum (essentially the imaginary part of the polarizability) within the random phase approximation (RPA) and the Bethe-Salpeter Equation (BSE), respectively. We note in passing that, due to the absence of long-range screening in (freestanding) 2D materials, the RPA spectrum in fact reduces to the non-interacting spectrum. ${ }^{5}$ The BSE calculations are performed using the PBE wave functions and energies with a scissors operator correction of the PBE band gap to match the $\mathrm{G}_{0} \mathrm{~W}_{0}$ gap. Spin-orbit coupling is fully included. For more details on the calculations we refer to Ref. ${ }^{26}$

In Fig. 11 we show, as an example, the optical absorbance spectrum of AsBrTe in the T-phase as calculated with the RPA and BSE methods, respectively. The absorbance, i.e. the percentage of incoming light absorbed by the monolayer, can be obtained from the 2D polarizability, $\alpha_{2 \mathrm{D}}$, according to $\operatorname{Abs}(\omega)=4 \pi \omega \alpha_{2 \mathrm{D}}(q \rightarrow$ $0, \omega) / c$, where $c$ is the speed of light. Due to the weak dielectric screening in (freestanding) 2D materials, the RPA spectrum resembles closely the single-particle result which reads

$$
\begin{aligned}
\alpha_{2 \mathrm{D}}(q \rightarrow 0, \omega)= & \frac{1}{N_{k} A \omega^{2}} \sum_{\mathbf{k} \in \mathrm{BZ}} \sum_{n}^{\text {occ. unocc. }} \sum_{n^{\prime}} \\
& \left|\left\langle\chi_{\mathbf{k} n}\left|\hat{p}_{x}\right| \chi_{\mathbf{k} n^{\prime}}\right\rangle\right|^{2} \delta\left(\omega-\varepsilon_{\mathbf{k} n}+\varepsilon_{\mathbf{k} n^{\prime}}\right),
\end{aligned}
$$

where $N_{k}$ is the number of $k$-points, $A$ is the area of the in-plane unit cell, $\chi$ denote spinor wave functions, and we have assumed normal incident light polarization along the $x$-axis. The BSE spectrum takes a similar form, except that the single-particle matrix elements and energy differences are replaced by their many-body analogues obtained by diagonalizing the twoparticle BSE Hamiltonian.

Above the direct quasiparticle (QP) gap of $2.64 \mathrm{eV}$ the RPA spectrum exhibits a number of peaks arising from groups of transitions in the QP band structure with similar energy. In- 

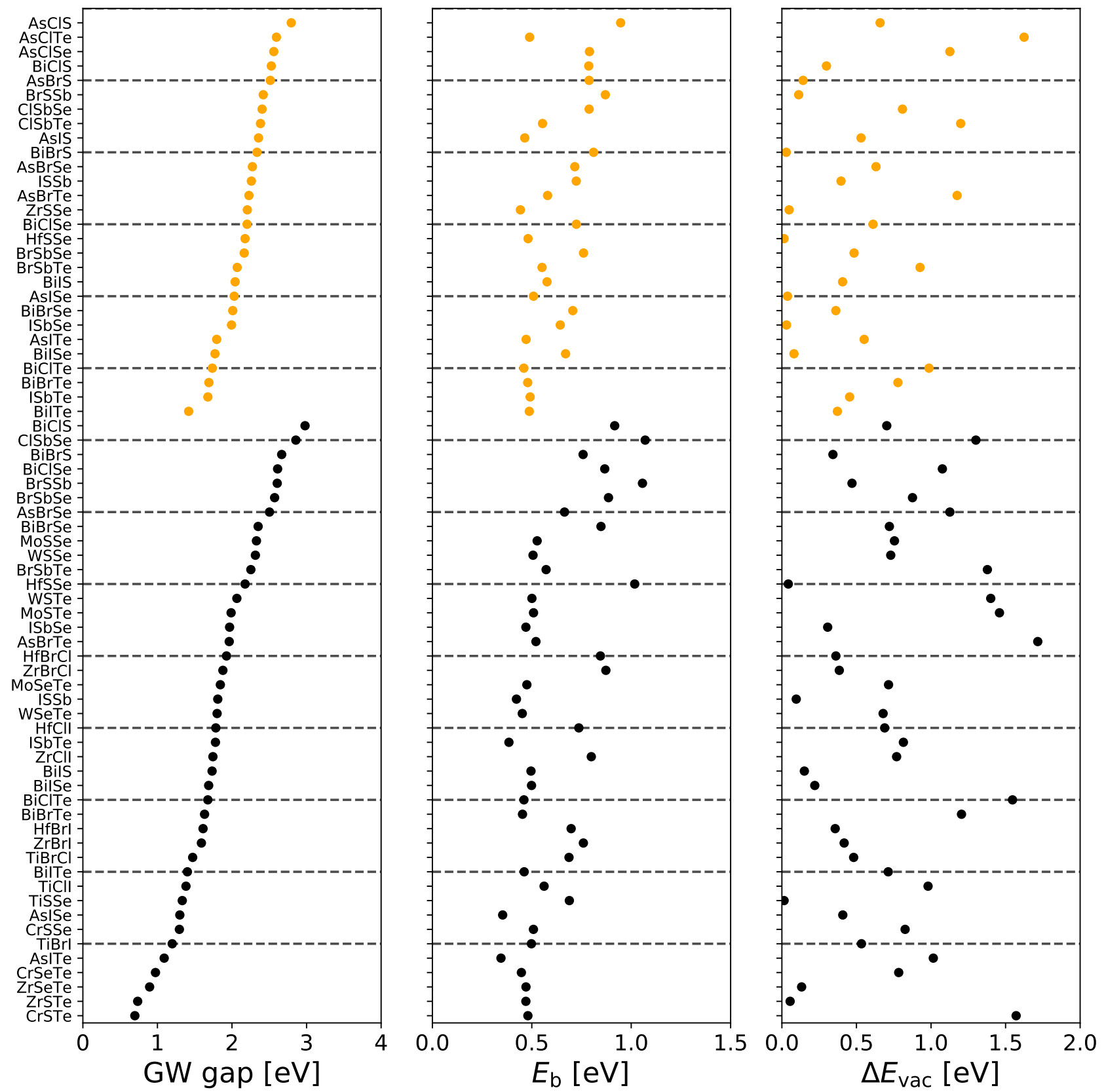

Figure 10: Band gaps (from $\mathrm{G}_{0} \mathrm{~W}_{0}$ ), exciton binding energies (from BSE), and vacuum level shifts across the monolayer for the 70 MXY Janus monolayers found to be stable, non-magnetic, and non-metallic. Results for the H-phase and T-phase are shown in black and orange, respectively. 


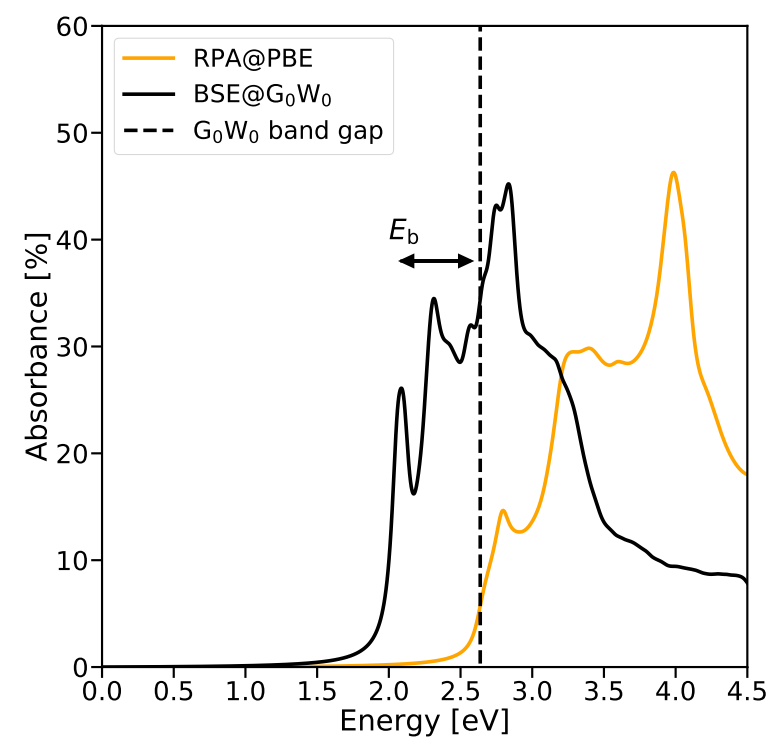

Figure 11: Optical absorbance of AsBrTe in the T-phase. The RPA (orange) is compared to the BSE spectrum that accounts for excitonic effects (black). The vertical line denotes the direct band gap obtained from $\mathrm{G}_{0} \mathrm{~W}_{0}$.

terestingly, the absorbance reaches $10 \%$ close to the band gap and exceeds $40 \%$ at $4 \mathrm{eV}$. These high absorption rates are a consequence of the $2 \mathrm{D}$ confinement and the similar orbital character of the conduction and valence bands (which both consists of mainly $\mathrm{Te}$ and $\mathrm{Br} p$ orbitals) giving rise to large oscillator strengths.

Compared to RPA, the BSE spectrum shows a distinct red shift of the excitation energies due to the attractive electron-hole interaction. In particular, strong excitonic peaks appear below the band gap with a binding energy of the lowest exciton of around $0.6 \mathrm{eV}$. The formation of a correlated excitonic state is associated with a significant increase in oscillator strength resulting in an absorbance exceeding 25\%, which dwarfs the absorbance strength of excitons in the TMDCs.

An overview of the exciton binding energies, defined as the difference between the direct QP band gap and the lowest eigenvalue of the BSE Hamiltonian, for the 70 semiconducting Janus monolayers can be seen in Fig. 10. The values range from $0.3 \mathrm{eV}$ and $1.0 \mathrm{eV}$ with a tendency of large exciton binding energies to be

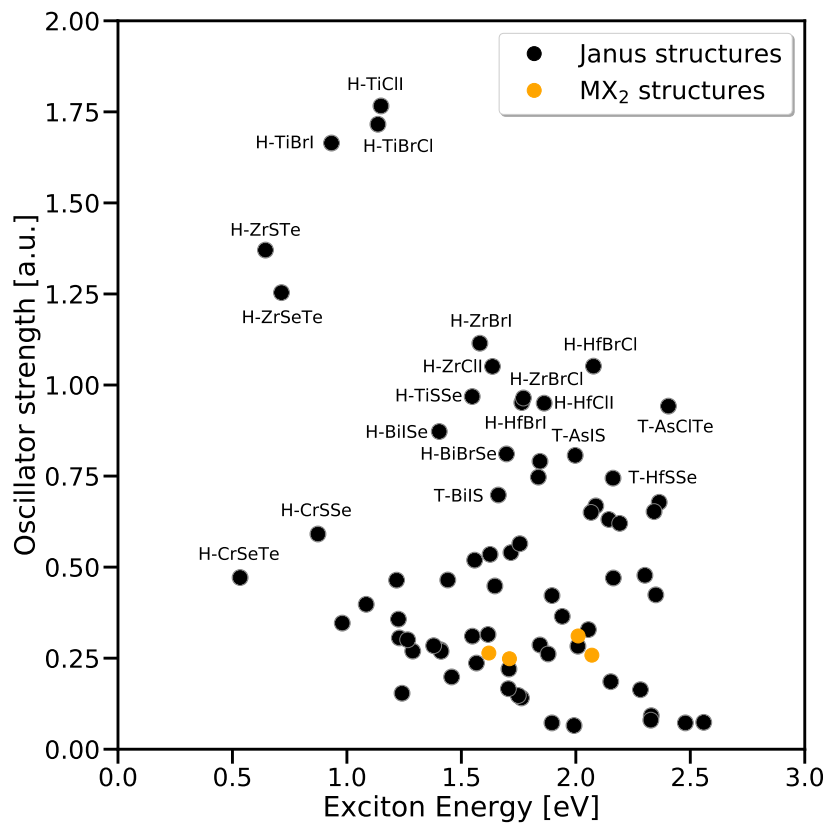

Figure 12: Oscillator strength of the lowest lying exciton peak as a function of the energy of the exciton energy. The oscillator strength has been normalized by the unit cell area. For reference the orange dots represent the well known TMDCs $\mathrm{MoS}_{2}, \mathrm{MoSe}_{2}, \mathrm{WS}_{2}$, and $\mathrm{WSe}_{2}$.

correlated with large QP band gaps. Such correlations have been observed before and can be explained by an approximate scaling of $E_{B}$ with the effective dielectric constant of the $2 \mathrm{D}$ material, which in turn is inversely proportional to the band gap. .46147

Finally, we consider the oscillator strengths of the lowest excitonic transitions. The oscillator strength is defined as $\left|\left\langle 0\left|\hat{p}_{x}\right| \Psi_{\text {ex }}\right\rangle\right|^{2}$, where $|0\rangle$ is the ground state, $\left|\Psi_{\text {ex }}\right\rangle$ is the lowest bright exciton, and $\hat{p}_{x}$ is the $x$ components of the momentum operator. In Fig. 12 the oscillator strength is plotted against the corresponding exciton energy for the 70 Janus monolayers. For reference the orange dots represent the well known TMDCs $\mathrm{MoS}_{2}, \mathrm{MoSe}_{2}, \mathrm{WS}_{2}$, and $\mathrm{WSe}_{2}$. For several of the Janus structures, the lowest exciton couples significantly stronger to light than is the case for the TMDCs. We find that strong exciton absorbance is most pronounced for Janus structures containing group IV transition metals ( $\mathrm{Ti}$ and $\mathrm{Zr}$ ). For these structures the valence and conduction bands are both located mainly on the $d$-orbitals of the transition 
metal - similar to the well known TMDCs. We ascribe the strong exciton absorbance in these materials to an unusually high joint density of states (JDOS) around the $\Gamma$-point of the BZ. In the group IV compounds, these transitions are located just above the direct band gap and leads to the formation of a highly localized (in real space) and strongly bound exciton. This is in contrast to group V and VI transition metal MXY and $\mathrm{MX}_{2}$ structures. For these structures we also find a high JDOS around the $\Gamma$-point but at energies much higher than the direct band gap leading to the formation of the wellknown strong C-exciton situated just above the band gap. We note that the extraordinary strong exciton oscillator strength observed for some of the Janus monolayers should not be directly attributed to the lack of mirror symmetry. Indeed, similar absorbance strengths are found for other $\mathrm{MX}_{2}$ structures containing, group IV transition metals such as $\mathrm{PtS}_{2}\left(\sec ^{\sqrt{26}}\right)$.

\section{Conclusions}

In summary, we have performed a systematic computational study of 216 MXY Janus monolayers lacking mirror symmetry. For the 70 most stable materials with a finite band gap, we performed detailed DFT and many-body calculations for the basic electronic and optical properties. The characterizing property of the Janus monolayers is the existence of a finite out-ofplane dipole moment giving rise to a difference in the electrostatic potential on the two sides of the layer. We found that these shifts can be substantial, reaching $1.8 \mathrm{eV}$ for some of the materials. We envision that such layers can be used to set up and control large out-of-plane electric fields inside van der Waals heterostructures. It was found that the band structures of the Janus monolayers can be qualitatively classified according to the groups of the metal (M) and non-metal (X,Y) atoms. While most combinations yield metallic compounds, the vast majority of the stable materials were found to be semiconducting. The band structures of the 70 most stable and semiconducting monolayers comprise both direct and indirect band gaps ranging from $0.7 \mathrm{eV}$ to $3.0 \mathrm{eV}$ (from $\mathrm{G}_{0} \mathrm{~W}_{0}$ calculations). A few compounds were found to exhibit Rashba-split conduction bands due to the combination of strong spin-orbit coupling and lack of mirror symmetry. As well known from other 2D semiconductors, the optical properties of the Janus monolayers are dominated by excitonic effects. The binding energies of the lowest excitons range from $0.3 \mathrm{eV}$ to $1.0 \mathrm{eV}$. Interestingly, the lowest excitons in Janus monolayers of group IV metal atoms ( $\mathrm{Ti}$ and $\mathrm{Zr}$ ) can exhibit extremely high oscillator strengths (almost ten times larger than those of the well known TMDCs) resulting in below-band gap absorbance peaks exceeding 40\%. All the data reported in this paper as well as data for other properties not discussed here, are available online as part of the Computational 2D Materials Database (C2DB).

\section{Methods}

The workflow used to calculate the properties of the Janus monolayers has been introduced in our previous work. ${ }^{26}$ It relies on the Atomic Simulation Environment (ASE) ${ }^{48}$ and the electronic structure code GPAW. $\stackrel{49}{ }$ The part of the workflow relevant for semiconducting materials, on which we focus in the present work, is shown in Fig. 13). In the first part of the workflow (left section) we relax the unit cell and atomic positions, check whether the $2 \mathrm{D}$ sheet has disintegrated during relaxation, and whether it is already contained in the database. Next, we classify the material according to crystal symmetries and occupied Wykoff sites, and calculate the heat of formation relative to the standard states of its elements and the energy above the convex hull, $E_{\text {hull }}$. The latter represents the energy relative to other competing bulk phases. In order to account for inaccuracies in the PBE total energy and possible substrate stabilizing effects, we consider materials to be thermodynamically stable if $E_{\text {hull }}<0.2 \mathrm{eV} /$ atom. We stress that a criterion of $E_{\text {hull }}<0.2 \mathrm{eV} /$ atom is rather conservative and will most likely generate false positives, i.e. materials that may not be stable or synthesisable in reality. ${ }^{50}$ On the 

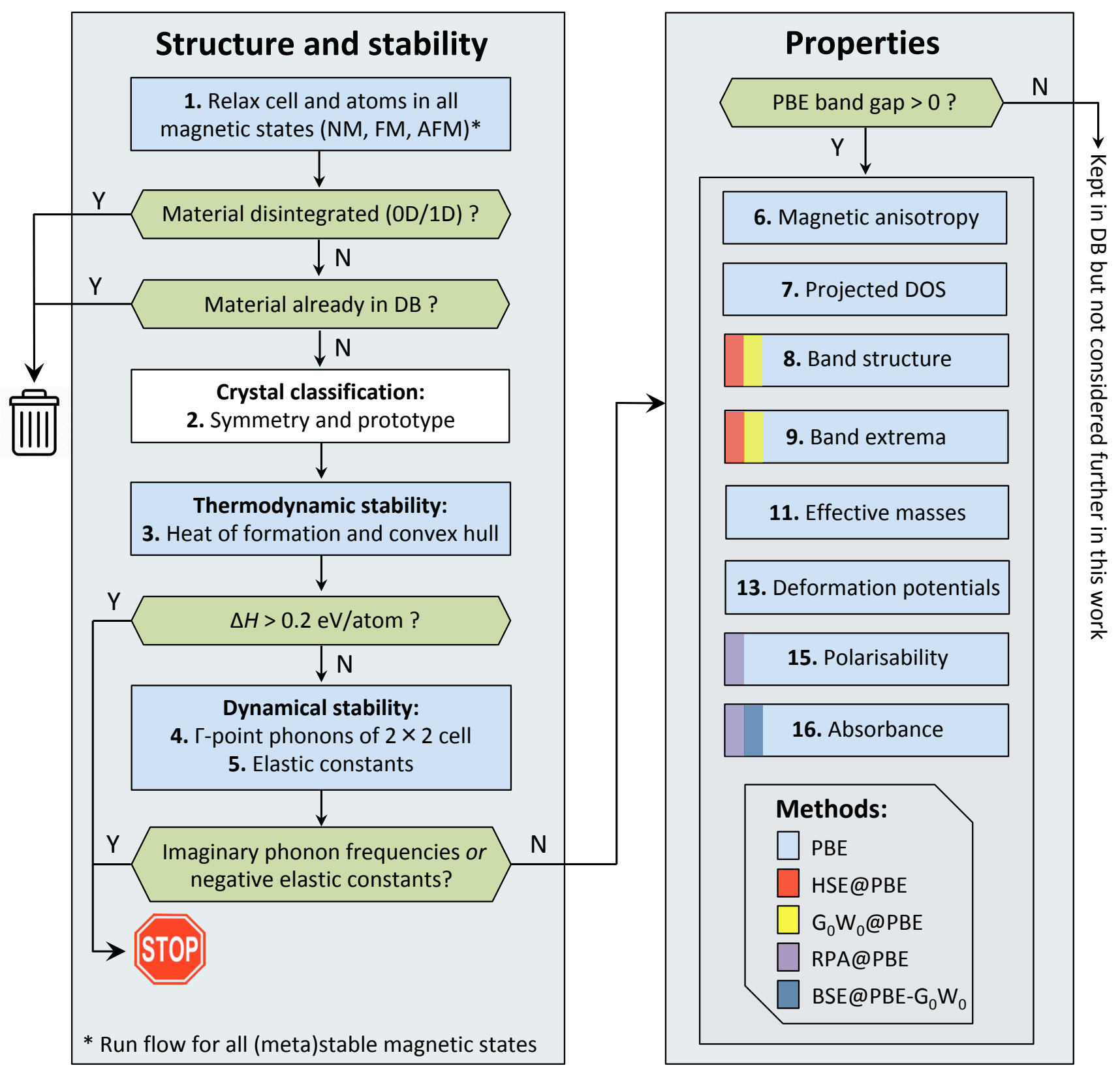

Figure 13: The workflow used to calculate the structure and properties of semiconducting Janus monolayers. Adapted with permission from Haastrup, S.; Strange, M.; Pandey, M.; Deilmann, T.; Schmidt, P. S.; Hinsche, N. F.; Gjerding, M. N.; Torelli, D.; Larsen, P. M.; Riis-Jensen, A. C.; Gath, J.; Jacobsen, K. W.; Mortensen, J. J.; Olsen, T.; Thygesen, K. S. 2D Mater. 2018, 5, 042002. Copyright 2018 IOP Publishing. 
other hand, with a more stringent stability criterion we run the risk of missing interesting candidates that might be stable in reality, e.g. due to the finite error bars on the DFT energies or possible stabilization via substrate interactions. We stress that the interested user can adjust the threshold value for $E_{\text {hull }}$ via the C2DB website. The dynamical stability is assessed by calculating the elastic tensor and phonon frequencies at high symmetry points of the Brillouin zone (BZ) (the $\Gamma$-point and the corners of the BZ). Materials with a negative stiffness coefficient or one or more imaginary phonon frequencies are deemed dynamically unstable and are not given further consideration.

In the second part of the workflow (right section) we calculate the band structure (at the $\mathrm{PBE}$, HSE, and $\mathrm{G}_{0} \mathrm{~W}_{0}$ level), the direct and indirect band gaps, the position of the band edges relative to the vacuum level and the corresponding $k$-points in the $\mathrm{BZ}$, the out-of-plane dipole moment, the effective masses, the polarizability (at the RPA@PBE level), and the optical absorption spectrum (at the $B S E @ G_{0} \mathrm{~W}_{0}$ level). We stress that the workflow calculates additional properties (projected density of states, Born charges, elastic tensor, deformation potentials, piezoelectric tensor, lattice contribution to the polarizability, and Raman spectrum). These properties are available in the C2DB but will not be discussed further in the present work. More details about the calculations including parameter settings can be found in Ref. 26

\section{Acknowledgement}

K.S.T. acknowledges funding from the European Research Council (ERC) under the European Union's Horizon 2020 research and innovation program (Grant Agreement No. 773122, LIMA).

\section{Supporting Information}

In tables I and II we provide some key electronic and optical properties for the 70 non-magnetic, stable, and semiconducting MXY Janus monolayers investigated in this work. The two tables report the properties for the T-phase and $\mathrm{H}$ phase structures, respectively. The Supporting Information is available free of charge on the ACS Publications website at DOI: link.

\section{References}

1. Mak, K. F.; Lee, C.; Hone, J.; Shan, J.; Heinz, T. F. Atomically Thin MoS2: a New Direct-Gap Semiconductor. Phys. Rev. Lett. 2010, 105, 136805.

2. Splendiani, A.; Sun, L.; Zhang, Y.; Li, T.; Kim, J.; Chim, C.-Y.; Galli, G.; Wang, F. Emerging Photoluminescence in Monolayer MoS2. Nano Lett. 2010, 10, 1271-1275.

3. Xiao, D.; Liu, G.-B.; Feng, W.; Xu, X.; Yao, W. Coupled Spin and Valley Physics in Monolayers of MoS2 and Other GroupVI Dichalcogenides. Phys. Rev. Lett. 2012, 108, 196802.

4. Jones, A. M.; Yu, H.; Ghimire, N. J.; Wu, S.; Aivazian, G.; Ross, J. S.; Zhao, B.; Yan, J.; Mandrus, D. G.; Xiao, D.; Yao, W.; $\mathrm{Xu}, \mathrm{X}$. Optical Generation of Excitonic Valley Coherence in Monolayer WSe2. Nat. Nano. 2013, 8, 634.

5. Thygesen, K. S. Calculating Excitons, Plasmons, and Quasiparticles in 2D Materials and van der Waals Heterostructures. 2D Mater. 2017, 4, 022004.

6. Bernardi, M.; Palummo, M.; Grossman, J. C. Extraordinary Sunlight Absorption and One Nanometer Thick Photovoltaics Using Two-Dimensional Monolayer Materials. Nano Lett. 2013, 13, 3664-3670.

7. Mouri, S.; Zhang, W.; Kozawa, D.; Miyauchi, Y.; Eda, G.; Matsuda, K. Thermal Dissociation of Inter-Layer Excitons in MoS2/MoSe2 Hetero-Bilayers. Nanoscale 2017, 9, 6674-6679.

8. Luong, D. H.; Lee, H. S.; Neupane, G. P.; Roy, S.; Ghimire, G.; Lee, J. H.; Vu, Q. A.; 
Lee, Y. H. Tunneling Photocurrent Assisted by Interlayer Excitons in Staggered van der Waals Hetero-Bilayers. Adv. Mater. 2017, 29, 1701512.

9. Wang, K.; Huang, B.; Tian, M.; Ceballos, F.; Lin, M.-W.; MahjouriSamani, M.; Boulesbaa, A.; Puretzky, A. A.; Rouleau, C. M.; Yoon, M.; Zhao, H.; Xiao, K.; Duscher, G.; Geohegan, D. B. Interlayer Coupling in Twisted WSe2/WS2 Bilayer Heterostructures Revealed by Optical Spectroscopy. ACS Nano 2016, 10, 6612-6622.

10. Ceballos, F.; Bellus, M. Z.; Chiu, H.-Y.; Zhao, H. Probing Charge Transfer Excitons in a MoSe2-WS2 van der Waals Heterostructure. Nanoscale 2015, 7, 1752317528 .

11. Ceballos, F.; Jung, M.; Lane, S. D.; Zeng, X. C.; Zhao, H. Highly Efficient and Anomalous Charge Transfer in van der Waals Trilayer Semiconductors. Nano. Lett. 2017, 17, 1623-1628.

12. Conley, H. J.; Wang, B.; Ziegler, J. I.; Haglund Jr, R. F.; Pantelides, S. T.; Bolotin, K. I. Bandgap Engineering of Strained Monolayer and Bilayer MoS2. Nano Lett. 2013, 13, 3626-3630.

13. Li, S.; Sun, M.; Chou, J.-P.; Wei, J.; Xing, H.; Hu, A. First-Principles Calculations of the Electronic Properties of SiCBased Bilayer and Trilayer Heterostructures. Phys. Chem. Chem. Phys. 2018, 20, 24726-24734.

14. Deilmann, T.; Thygesen, K. S. Interlayer Excitons with Large Optical Amplitudes in Layered van der Waals Materials. Nano Lett. 2018, 18, 2984-2989.

15. Fei, Z.; Rodin, A.; Andreev, G. O.; Bao, W.; McLeod, A.; Wagner, M.; Zhang, L.; Zhao, Z.; Thiemens, M.; Dominguez, G.; Fogler, M. M.; Castro Neto, A. H.; Lau, C. N.; Keilmann, F.; Basov, D. N. Gate-Tuning of
Graphene Plasmons Revealed by Infrared Nano-Imaging. Nature 2012, 487, 82.

16. Qiu, Z.; Trushin, M.; Fang, H.; Verzhbitskiy, I.; Gao, S.; Laksono, E.; Yang, M.; Lyu, P.; Li, J.; Su, J.; Telychko, M.; Watanabe, K.; Taniguchi, T.; Wu, J.; Castro Neto, Y. L., A H; Eda, G.; Adam, S.; Lu, J. Giant Gate-Tunable Bandgap Renormalization and Excitonic Effects in a 2D Semiconductor. Sci. Adv. 2019, 5, eaaw2347.

17. Latini, S.; Olsen, T.; Thygesen, K. S. Excitons in van der Waals Heterostructures: The Important Role of Dielectric Screening. Phys. Rev. B: Condens. Matter Mater. Phys. 2015, 92, 245123.

18. Chernikov, A.; Berkelbach, T. C.; Hill, H. M.; Rigosi, A.; Li, Y.; Aslan, O. B.; Reichman, D. R.; Hybertsen, M. S.; Heinz, T. F. Exciton Binding Energy and Nonhydrogenic Rydberg Series in Monolayer WS2. Phys. Rev. Lett. 2014, 113, 076802.

19. Geim, A. K.; Grigorieva, I. V. Van der Waals Heterostructures. Nature 2013, 499, 419.

20. Cui, X.; Lee, G.-H.; Kim, Y. D.; Arefe, G.; Huang, P. Y.; Lee, C.-H.; Chenet, D. A.; Zhang, X.; Wang, L.; Ye, F.; Pizzocchero, F.; Jessen, B. S.; Watanabe, K.; Taniguchi, T.; Muller, D. A.; Low, T.; Hone, J. Multi-Terminal Transport Measurements of MoS2 Using a van der Waals Heterostructure Device Platform. Nat. Nano. 2015, 10, 534.

21. Naguib, M.; Mochalin, V. N.; Barsoum, M. W.; Gogotsi, Y. 25th Anniversary Article: MXenes: a New Family of TwoDimensional Materials. Adv. Mater. 2014, 26, 992-1005.

22. Huang, B.; Clark, G.; NavarroMoratalla, E.; Klein, D. R.; Cheng, R.; Seyler, K. L.; Zhong, D.; Schmidgall, E.; McGuire, M. A.; Cobden, D. H.; Yao, W.; 
Xiao, D.; Jarillo-Herrero, P.; Xu, X. Layer-Dependent Ferromagnetism in a van der Waals Crystal Down to the Monolayer Limit. Nature 2017, 546, 270.

23. Lu, A.-Y.; Zhu, H.; Xiao, J.; Chuu, C.P.; Han, Y.; Chiu, M.-H.; Cheng, C.C.; Yang, C.-W.; Wei, K.-H.; Yang, Y.; Wang, Y.; Sokaras, D.; Nordlund, D.; Yang, P.; Muller, D. A.; Chou, M.-Y.; Zhang, X.; Li, L.-J. Janus Monolayers of Transition Metal Dichalcogenides. Nat. Nanotechnol. 2017, 12, 744-749.

24. Zhang, J.; Jia, S.; Kholmanov, I.; Dong, L.; Er, D.; Chen, W.; Guo, H.; Jin, Z.; Shenoy, V. B.; Shi, L.; Lou, J. Janus Monolayer Transition-Metal Dichalcogenides. ACS Nano 2017, 11, 8192-8198.

25. Fülöp, B.; Tajkov, Z.; Pető, J.; Kun, P.; Koltai, J.; Oroszlány, L.; Tóvári, E.; Murakawa, H.; Tokura, Y.; Bordács, S.; Tapasztó, L.; Csonka, S. Exfoliation of Single Layer BiTeI Flakes. 2D Mater. 2018, 5, 031013.

26. Haastrup, S.; Strange, M.; Pandey, M.; Deilmann, T.; Schmidt, P. S.; Hinsche, N. F.; Gjerding, M. N.; Torelli, D.; Larsen, P. M.; Riis-Jensen, A. C.; Gath, J.; Jacobsen, K. W.; Mortensen, J. J.; Olsen, T.; Thygesen, K. S. The Computational 2D Materials Database: HighThroughput Modeling and Discovery of Atomically Thin Crystals. 2D Mater. 2018, 5, 042002 .

27. Lebègue, S.; Björkman, T.; Klintenberg, M.; Nieminen, R. M.; Eriksson, O. Two-Dimensional Materials from Data Filtering and $A b$ Initio Calculations. Phys. Rev. X 2013, 3, 031002.

28. Ashton, M.; Paul, J.; Sinnott, S. B.; Hennig, R. G. Topology-Scaling Identification of Layered Solids and Stable Exfoliated 2D Mater. Phys. Rev. Lett. 2017, 118, 106101.

29. Mounet, N.; Gibertini, M.; Schwaller, P.; Campi, D.; Merkys, A.; Marrazzo, A.; Sohier, T.; Castelli, I. E.; Cepellotti, A.;
Pizzi, G.; Marzari, N. Two-Dimensional Materials from High-Throughput Computational Exfoliation of Experimentally Known Compounds. Nat. Nano. 2018, 13, 246.

30. Nakagawa, N.; Hwang, H. Y.; Muller, D. A. Why Some Interfaces Cannot Be Sharp. Nat. Mater. 2006, 5, 204.

31. Nazir, S.; Cheng, J.; Yang, K. Creating Two-Dimensional Electron Gas in Nonpolar Oxide Interface via Polarization Discontinuity: First-Principles Analysis of $\mathrm{CaZrO} 3 / \mathrm{SrTiO} 3$ Heterostructure. ACS Appl. Mater. Interfaces 2015, 8, 390399.

32. Wallace, S. K.; Svane, K. L.; Huhn, W. P.; Zhu, T.; Mitzi, D. B.; Blum, V.; Walsh, A. Candidate Photoferroic Absorber Materials for Thin-Film Solar Cells from Naturally Occurring Minerals: Enargite, Stephanite, and Bournonite. Sustainable Energy Fuels 2017, 1, 1339-1350.

33. Butler, K. T.; Frost, J. M.; Walsh, A. Ferroelectric Materials for Solar Energy Conversion: Photoferroics Revisited. Energy Environ. Sci. 2015, 8, 838-848.

34. Sun, Y.; Shuai, Z.; Wang, D. Janus Monolayer of WSeTe, a New Structural Phase Transition Material Driven by Electrostatic Gating. Nanoscale 2018, 10, 21629-21633.

35. Ma, X.; Wu, X.; Wang, H.; Wang, Y. A Janus MoSSe Monolayer: a Potential Wide Solar-Spectrum Water-Splitting Photocatalyst with a Low Carrier Recombination Rate. J. Mater. Chem. A 2018, 6, 22952301.

36. Dong, L.; Lou, J.; Shenoy, V. B. Large In-Plane and Vertical Piezoelectricity in Janus Transition Metal Dichalchogenides. ACS Nano 2017, 11, 8242-8248.

37. Riis-Jensen, A. C.; Pandey, M.; Thygesen, K. S. Efficient Charge Separation in 2D 
Janus van der Waals Structures with BuiltIn Electric Fields and Intrinsic $p$-n Doping. J. Phys. Chem. C 2018, 122, 24520-24526.

38. Palsgaard, M.; Gunst, T.; Markussen, T.; Thygesen, K. S.; Brandbyge, M. Stacked Janus Device Concepts: Abrupt pnJunctions and Cross-Plane Channels. Nano Lett. 2018, 18, 7275-7281.

39. Cavalcante, L. S.; Gjerding, M. N.; Chaves, A.; Thygesen, K. S. Enhancing and Controlling Plasmons in Janus MoSSe-Graphene Based Van Der Waals Heterostructures. J. Phys. Chem. C 2019,

40. Saal, J. E.; Kirklin, S.; Aykol, M.; Meredig, B.; Wolverton, C. Materials Design and Discovery with High-Throughput Density Functional Theory: The Open Quantum Materials Database (OQMD). Jom 2013, 65, 1501-1509.

41. Bahramy, M.; Arita, R.; Nagaosa, N. Origin of Giant Bulk Rashba Splitting: Application to BiTeI. Phys. Rev. B: Condens. Matter Mater. Phys. 2011, 84, 041202.

42. Cheng, Y.; Zhu, Z.; Tahir, M.; Schwingenschlögl, U. Spin-Orbit-Induced Spin Splittings in Polar Transition Metal Dichalcogenide Monolayers. Europhys. Lett. 2013, 102, 57001 .

43. Ugeda, M. M.; Bradley, A. J.; Shi, S.F.; Felipe, H.; Zhang, Y.; Qiu, D. Y.; Ruan, W.; Mo, S.-K.; Hussain, Z.; Shen, Z.X.; Wang, F.; Louie, S. G.; Crommie, M. F. Giant Bandgap Renormalization and Excitonic Effects in a Monolayer Transition Metal Dichalcogenide Semiconductor. Nat. Mater. 2014, 13, 1091.

44. Britnell, L.; Ribeiro, R.; Eckmann, A.; Jalil, R.; Belle, B.; Mishchenko, A.; Kim, Y.-J.; Gorbachev, R.; Georgiou, T.; Morozov, S.; Grigorenko, G. A. K. C. C., A N; Castro Neto, A. H.; Novoselov, K. S. Strong Light-Matter Interactions in Heterostructures of Atomically Thin Films. Science 2013, 340, 1311-1314.
45. Schaibley, J. R.; Yu, H.; Clark, G.; Rivera, P.; Ross, J. S.; Seyler, K. L.; Yao, W.; Xu, X. Valleytronics in 2D materials. Nat. Rev. Mater. 2016, 1, 16055.

46. Olsen, T.; Latini, S.; Rasmussen, F.; Thygesen, K. S. Simple Screened Hydrogen Model of Excitons in Two-Dimensional Materials. Phys. Rev. Lett. 2016, 116, 056401.

47. Jiang, Z.; Liu, Z.; Li, Y.; Duan, W. Scaling Universality Between Band Gap and Exciton Binding Energy of Two-Dimensional Semiconductors. Phys. Rev. Lett. 2017, $118,266401$.

48. Larsen, A. H.; Mortensen, J. J.; Blomqvist, J.; Castelli, I. E.; Christensen, R.; Dułak, M.; Friis, J.; Groves, M. N.; Hammer, B.; Hargus, C.; Hermes, E. D.; Jennings, P. C.; Jensen, P. B.; Kermode, J.; Kitchin, J. R.; Kolsbjerg, E. L.; Kubal, J.; Kaasbjerg, K.; Lysgaard, S.; Maronsson, J. B. et al. The Atomic Simulation Environment-A Python Library for Working with Atoms. J. Phys.: Condens. Matter 2017, 29, 273002.

49. Enkovaara, J.; Rostgaard, C.; Mortensen, J. J.; Chen, J.; Dułak, M.; Ferrighi, L.; Gavnholt, J.; Glinsvad, C.; Haikola, V.; Hansen, H. A.; Kristoffersen, H. H.; Kuisma, M.; Larsen, A. H.; Lehtovaara, L.; Ljungberg, M.; LopezAcevedo, O.; Moses, P. G.; Ojanen, J.; Olsen, T.; Petzold, V. et al. Electronic Structure Calculations with GPAW: A Real-Space Implementation of the Projector Augmented-Wave Method. J. Phys.: Condens. Matter 2010, 22, 253202.

50. Malyi, O. I.; Sopiha, K. V.; Persson, C. Energy, Phonon, and Dynamic Stability Criteria of Two-Dimensional Materials. ACS Appl. Mater. Interfaces 2019, 